Impurity concentration determination in 6H-SiC

A. Henry, O. Kordina, C. Hallin,

C. Hemmingsson and E. Janzén

Department of Physics and Measurement Technology, Linköping University, S-581 83 Linköping, Sweden.

The intensity ratio between intrinsic and extrinsic components in the photoluminescence (PL) spectra reflects the impurity concentration. This behaviour has previously been used for impurity concentration calibration in various semiconductors such as $\mathrm{Si}, \mathrm{GaAs}, \mathrm{InP}$ and diamond. It was recently proposed to be applicable in the case of the nitrogen donors in $6 \mathrm{H}-\mathrm{SiC}$ [1]. However we will show in this paper that careful experimental conditions must be chosen in this case. The investigated samples were grown by the chemical vapour deposition technique and covered the range of net impurity concentration of $1 \times 10^{14}$ to $1 \times 10^{17}$ $\mathrm{cm}^{-3}$. N-type, p-type, uncompensated and compensated materials were used. The PL spectra were recorded in the near band gap emission of the $6 \mathrm{H}-\mathrm{SiC}$ at various temperatures between $4.2 \mathrm{~K}$ and $35 \mathrm{~K}$. The intensity ratio between the nitrogen bound exciton (BE) and the free exciton (FE) is shown to be dependent on the excitation power and the temperature used during the PL experiments and also on the the degree of compensation.

[1] L.L Clemen, M. Yoganathan, W.J. Choyke, R.P. Devaty, H.S. Kong, J.A. Edmond, D.J. Larkin, J.A. Powell and A.A. Burk jr, presented at the ICSCRM, nov 1-3 1993, Washington DC

\section{Far-infrared Magneto-optical Studies of D- Band in Doped GaAs-AIGaAs Multiple Quantum Well Structures}

Y.H.Chang, C.H.Lee.H.Y.Hao, Y.M.Sheu, andC.D.Hu Department of Physics. Narional Taiwan University, Taipei, Taiwan 107.Republic of China H.H. Lin

Department of Electrical Engineering, National Taiwan University, Taipei, Taiwan 107. Republic of China

$D^{*}$ ion, a shallow donor which binds two electrons and is negatively charged, could be found in GaAs quantum well (QW) when both the QW and the potential barrier are doped with n-type impurities. As the $\mathrm{D}^{-}$concentration in $\mathrm{OW}$ increases, it can be expected that $\mathrm{D}^{\circ}$ level will broaden into a $\mathrm{D}^{-}$band, which is also known as the upper Hubbard band. In this paper we report far infrared magneto-optical studies of the properties of $\mathrm{D}^{-}$band. The samples used in this study are $\mathrm{GaAs} / \mathrm{Al}_{3} \mathrm{Ga}_{7} \mathrm{As}$ multiple $\mathrm{QW}$ structures grown by molecular beam epitaxy. The width of the GaAs OWs are $200 \dot{A}$ and the width of the AlGaAs potential barriers are $600 \dot{A}$. All samples are $\delta$-doped at the center of the QWs and at the center of the potential barriers. The doping concentration in the wells and in the barriers are the same and are $2 * 10^{10} \mathrm{~cm}^{-2}, 4^{*} 10^{10} \mathrm{~cm}^{-2}, 8 * 10^{10} \mathrm{~cm}^{2}$, and $16^{*} 10^{10} \mathrm{~cm}^{-2}$, respectively. Far-infrared magneto-transmission measurements were performed using a slow scan Fourier transform spectrometer in conjunction with a 9T superconducting magnet. At high magnetic fields and $T=5 \mathrm{~K}$, three-peak structures are observed for the sample with lowest doping concentration. These three peaks are attributed to electron cyclotron resonance (CR), intra- $D^{\circ}$ ( $\left(\mathrm{s}-\mathrm{s}^{-}>2 \mathrm{p}^{+}\right)$transition and intra-D $\left(1 s^{2}->1 s^{5} 2 p^{+}\right)$transition, respectively. As the impurity concentration increases, intra- $D^{\alpha}$ transition gradually shifts to lower energy, loses it's absorption intensity and finally merges with intra-D transition. In the temperature dependent studies, different behavior are observed for samples with different doping concentration at high magnetic fields. For the sample with doping concentration of $4 * 10^{10}$ $\mathrm{cm}^{-2}$, as the temperature increases, intra- $\mathrm{D}^{-}$transition loses it's absorption intensity while the absorption intensities of CR and intra$D^{0}$ transition increase. This behavior is consistent with a model calculation which treats $\mathrm{D}^{\prime}$ and $\mathbf{D}^{-}$as isolated centers with welldefined binding energies. For the sample with doping concentration of $16^{*} 10^{10} \mathrm{~cm}^{-2}$, between $\mathrm{T}=5 \mathrm{~K}$ and $\mathrm{T}=30 \mathrm{~K}$, intra- $D^{\circ}$ transition could not be identified, the spectra are dominated by intra- $D$ transitions. A D band with broad bandwidth is needed to understand such a behavior. We'll also discuss the significance of our experimental results on the interpretation of metal-insulator transitions in doped semiconductor systems.

This work is supported by the National Science Council of the Republic of China

\section{Doping Efficiency and Compensation} Mechanism in N-Doped $\mathrm{ZnSe}$

$$
\begin{gathered}
\text { Byoung-Ho Cheong, }{ }^{\dagger} \text { C. H. Park, }{ }^{*} \text { and } \\
\text { K. J. Chang }{ }^{\dagger}
\end{gathered}
$$

$\uparrow$ Department of Physics, Korea Advanced Institute of Science and Technology,

373-1 Kusung-dong, Yusung-ku, Taejon, Korea

* NEC Research Institute, 4 Independence Way, Princeton, NJ 08540, U.S.A

After the success of low resistance $p$-type doping in $\mathrm{ZnSe}$ using a radical $N$ source, there is growing interest in the doping efficiency of $\mathrm{N}$ doped $\mathrm{ZnSe}$ because of its applications for bluegreen laser devices. However, when the $\mathrm{N}$ concentration exceeds above $10^{18} \mathrm{~cm}^{-3}$, a doping efficiency abruptly decreases, showing strong selfcompensation.

Through the first-principles pseudopotential method, we calculate the formation energies for various $\mathrm{N}$-related defects including native ones and find that a compensation effect of $\mathrm{N}-\mathrm{N}$ complexes on acceptors is very significant in highly $\mathrm{N}$-doped $\mathrm{ZnSe}$. We find that a substitutional acceptor $\mathrm{N}_{S e}^{-}$is most stable among the defects, while the broken-bonding configuration with $\mathrm{C}_{3 v}$ symmetry is a metastable state against the $T_{d^{-}}$ symmetry structure. The estimated maximum acceptor density is about $10^{18} \mathrm{~cm}^{-3}$ under Se-rich condition, in good agreement with experiments. However, for $\mathrm{N}$ concentraions above $10^{18} \mathrm{~cm}^{-3}$, the neutral $\mathrm{N}_{2}$ molecule at an interstitial site is energetically favorable and neutralizes the acceptor activity, lowering doping efficiency. Under $\mathrm{Zn}$-rich condition, since the $\mathrm{N}$ atom prefers to occupying the Se sublattice as a substitutional, the maximum carrier concentration is increased up to about $10^{19} \mathrm{~cm}^{-3}$, giving rise to higher doping efficiency. However, the acceptors are still compensated by the [100]-split interstitial N-N complexes which behave as a double donor at higher $\mathrm{N}$ concentrations.

\section{DONOR PROPERTIES OF SC IMPURITY IN CdTE AND ZnTE CRYSTALS}

\author{
Yu.P.Gnatenko, P.N.Bukivski, \\ R.V.Gamernik, I.O.Farina
}

Institute of Physics, National Academy of Sciences, 252022 , Kiev-22, pr.Nauki ,46, UKRAINE

Studing low-temperature ex- 